\title{
Preface to the Special Issue on Environmental Catalysis and Materials
}

Catalysis has an important role to play in environment remediation and the generation of clean energy. Due to the rapid development of industrial activities in the past decades, pollution and energy shortage are issues that threaten human health and social sustainability. Among the known strategies to tackle these problems, the catalytic approach has attracted more attention due to its advantages such as (1) the facile and complete decomposition of nearly all kinds of organic pollutants, (2) no production of secondary contaminants, (3) gives environment-benign and atom-economy processes, and (4) green production of energy. The core issue in a successful catalytic process is the development of a suitable catalyst that is highly efficient.

Heterogeneous catalysts that are easily separable from the reaction system are desired in applications. The activity of a catalyst is decided by factors such as the chemical composition, phase structure, surface properties, pore structure and morphology. In the past years, much effort have been put into the construction of novel catalysts that are well-defined in structure, high in specific surface area and rich in surface defects. However, in industrial applications, heterogeneous catalysts are less successful than homogeneous catalysts because the former have much less exposed active sites than the latter. In addition, the nature of the active sites and the mechanism of the catalytic processes have still to be clarified through the use of in situ characterization techniques.

This special issue of Chinese Journal of Catalysis contains 5 reviews and 15 research articles that focus on the recent advances in catalysis for pollutant removal and green synthesis. We hope that this Special Issue will be a valuable reference for the design of efficient catalysts and the development of catalytic processes for new applications.

We thank all authors and reviewers for their expertise and the editorial and production staff for their time and effort. It is because of their hard work that this Special Issue is now being published.

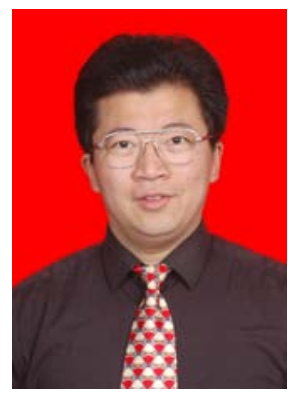

Prof. Shuang-Feng Yin (Guest Editor) State Key Laboratory of Chemo/Biosensing and Chemometrics College of Chemistry and Chemical Engineering Hunan University E-mail: sf_yin@hnu.edu.cn

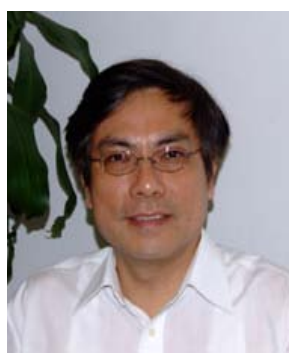

Prof. Chak-Tong Au (Guest Editor)

Department of Chemistry

College of Chemistry and Chemical Engineering

Hunan Institute of Engineering

E-mail: pctau@hkbu.edu.hk 